\title{
hs-cTnT measurement to rule out MI
}

A single-centre study conducted in Sweden has demonstrated that measurement of cardiac troponin T level using a highsensitivity assay (hs-cTnT) could rule out myocardial infarction (MI) in patients with chest pain, without the need for serial biomarker measurement. "We believe that hospital admission could have been avoided in the majority of these patients," write the authors of the paper, published in JACC. "The potential savings for health-care providers are enormous."

In their study, Bandstein et al. included all patients aged $\geq 25$ years who presented with chest pain to the emergency department (ED) at Karolinska University Hospital in Stockholm between December 2010 and December 2012, and who had their hs-cTnT level measured at least once $(n=14,636)$. Of these individuals, 8,907 (61\%) had an initial hs-cTnT level $<5 \mathrm{ng} / \mathrm{l}$, and $21 \%$ of these patients were hospitalized. Only 39 (0.44\%) patients with a hs-cTnT level $<5 \mathrm{ng} / \mathrm{l}$ were diagnosed with a nonfatal, type $1 \mathrm{MI}$ within 30 days; 15 of these individuals $(0.17 \%)$ had no electrocardiographic changes on their first presentation to the ED.

The negative predictive value for nonfatal MI of hs-cTnT level $<5 \mathrm{ng} / 1$ plus no electrocardiographic signs of ischaemia was $99.8 \%$ (95\% CI 99.799.9\%), and the negative predictive value for death within 30 days was 100\% (95\% CI 99.9-100\%). After multivariable adjustment, the risk of death at 1 year among patients with an initial hs-cTnT level $<5 \mathrm{ng} / \mathrm{l}$ did not differ between those admitted to hospital and those who were not admitted.

"As chest pain is one of the most common symptoms in patients seeking medical attention in the ED, our strategy may help to reduce overcrowding of the ED," conclude the authors.

\section{Alexandra Roberts}

Original article Bandstein, N. et al. Undetectable high sensitivity cardiac troponin T level in the emergency department and risk of myocardial infarction. J. Am. Coll. Cardiol. doi:10.1016/j.jacc.2014.03.017 